\title{
Enseñanza del Análisis Sensorial en un Panel de Cata y su relación con las propiedades del Aceite de Oliva Virgen
}

\author{
Vanessa Martos Núñez - Universidad de Granada \\ Celia Monteagudo González - Universidad de Granada \\ Ali Ahmad - Universidad de Granada \\ Mํㅡㄴ Luisa Lorenzo Tovar - Universidad de Granada
}

Recepción: 27.12.2021 | Aceptado: 25.01.2022

Correspondencia a través de ORCID: Vanessa Martos

\author{
0000-0001-6442-7968 \\ (D) $0000-0003-0529-379 \mathrm{X}$ \\ (D) $0000-0001-5530-7374$ \\ (i) $0000-0002-5997-4745$
}

Citar: Martos Núñez, V, Monteagudo González, C, Ahmad, A y Lorenzo Tovar, ML (2022). Enseñanza del Análisis Sensorial en un Panel de Cata y su relación con las propiedades del Aceite de Oliva Virgen. REIDOCREA, 11(7), 87-89.

Área o categoría del conocimiento: Innovación docente

Resumen: La valoración organoléptica, se puede llevar a cabo científicamente mediante ensayos sensoriales realizados por un panel analítico y aplicando un tratamiento estadístico para analizar los resultados y conocer la calidad intrínseca del alimento, los catadores han de ser entrenados y esto constituye un aprendizaje en análisis sensorial, actividad de mucho futuro profesional hoy en día. El panel analítico está constituido por 8/12 catadores seleccionados y entrenados dirigidos por un Jefe de Panel, que posee conocimientos y formación para la organización y realización de los ensayos, siguiendo el método de valoración organoléptica del Consejo Oleícola Internacional (COI). Este método tiene por finalidad, establecer los criterios necesarios para valorar las características del flavor del Aceite de Oliva Virgen (AOV) y desarrollar la metodología para su clasificación, en función de la intensidad de los defectos y la existencia de atributo frutado. Se entiende por flavor el conjunto de percepciones de estímulos olfatogustativos, táctiles y quinestésicos que permiten a un sujeto identificar un alimento y establecer un criterio, a distintos niveles de agrado o desagrado. Al realizar la cata del AOV, hay que distinguir entre atributos positivos y negativos de este, entendiéndose por atributo la propiedad característica perceptible.

Palabra clave: Análisis sensorial

Teaching Sensory Analysis in a Tasting Panel and its relation to the properties of Virgin Olive Oil

Abstract: The organoleptic evaluation can be carried out scientifically through sensory tests performed by an analytical panel and applying a statistical treatment to analyze the results and know the intrinsic quality of the food, the tasters have to be trained and this is an apprenticeship in sensory analysis, an activity of great professional future nowadays. The analytical panel is made up of $8 / 12$ selected and trained tasters directed by a Panel Leader, who has the knowledge and training to organize and carry out the tests, following the organoleptic assessment method of the International Olive Council (IOC). The purpose of this method is to establish the necessary criteria to evaluate the flavor characteristics of Virgin Olive Oil (VOO) and to develop the methodology for its classification, according to the intensity of the defects and the existence of the fruity attribute. Flavor is understood as the set of perceptions of olfactory-gustatory, tactile and kinesthetic stimuli that allow a subject to identify a food and establish a criterion, at different levels of liking or disliking. When tasting the AOV, it is necessary to distinguish between its positive and negative attributes, whereby attribute is understood as the perceptible characteristic property.

Keyword: Sensory Analysis

\section{Introducción}

En la actualidad existe una preocupación en relación con la seguridad y calidad de los productos agroalimentarios, debido, al impacto que la alimentación ejerce en la salud y bienestar, esto ha provocado que el mercado exija mayores controles y exámenes que garanticen dicha seguridad y calidad a lo largo de la cadena alimentaria, y se determine un exhaustivo seguimiento de la trazabilidad. 
Los análisis sensoriales (paneles de cata) constituyen un factor clave en cualquier estudio y análisis de evaluación de la conformidad, especialmente en el campo agroalimentario (UNE EN-ISO/IEC 17025).

El Seminario de Estudios Gastronómicos y Enológicos de la UGR (SEGE), de la Universidad de Granada, tiene un Panel de multidisciplinar de Cata analítico, para realizar evaluaciones sensoriales de diversos tipos de productos agroalimentarios, formado por catadores oficiales evaluados y entrenados junto a un jefe de panel, realizando pruebas sensoriales de múltiples alimentos, actualmente este Panel de Cata desarrolla el Proyecto H2020 titulado VIRTUOUS: (Virtual tongue to predlct the oRganoleptic profile of mediterranean IngredienTs and their effect on hUman hOmeostasi by means of an integrated compUtational multiphysic Splatform) que une las Ciencias Agroalimentarias y la Inteligencia Artificial para crear una 'lengua virtual' a través de un marco computacional integrado, capaz de detectar alimentos para ligandos naturales dirigidos a receptores gustativos. La idea es concebir un predictor del sabor que se aplique a los productos alimentarios europeos, algo que sin duda impulsará y ampliará el mercado alimentario europeo a nivel mundial (Pallante L. et al. 2021).

El algoritmo inteligente propuesto en VIRTUOUS, mediante la integración de técnicas de descubrimiento de medicamentos y algoritmos para grandes datos, predecirá el perfil organoléptico de un alimento específico basado en su composición química.

También se puede aplicar esta plataforma de inteligencia artificial que aporta VIRTUOUS para crear una "herramienta de diseño asistida por ordenador" para la tecnología alimentaria de la UE. Por ejemplo, basándose en la predicción del sabor, la plataforma VIRTUOUS puede servir en el futuro para predecir los resultados de un injerto de uva específico. VIRTUOUS también puede combinarse con otras tecnologías para mejorar la agricultura de precisión.

\section{Objetivos}

El objetivo de este trabajo es dar las nociones para formar a estudiantes de Ciencias agroalimentarias y profesionales del sector, en el método científico del análisis sensorial para analizar, identificar, caracterizar, valorar organolépticamente y comprender las características organolépticas de productos agroalimentarios de interés socioeconómico tales como aceites, lácteos, panes, aguas, chacinas y vinos, así como otros productos y medios de la producción agraria generados principalmente en la provincia de Granada y en el resto de Andalucía, y de interés en toda la Cuenca Mediterránea. El objetivo del Panel de Cata de la Universidad de Granada, es ofrecer una herramienta de utilidad para el análisis y estudio de nuestro patrimonio, especialmente del gastronómico y enológico.

\section{Método}

Los catadores guiados por un jefe de panel, en grupos de 12 personas, han de ser entrenados y formados en la disciplina del análisis sensorial valorando diversos productos semanalmente con el objetivo de saber identificar todas las características organolépticas y sensoriales. Para ello se aplica la metodología seguida en reglamento (UE) No 1169/2011 del parlamento europeo, y en el caso concreto del aceite de oliva se sigue el Reglamento (CE) 2568/1991, referido a las características de los aceites de oliva y de los aceites de orujo y sobre sus métodos de análisis.

El Panel de Catadores de Aceite de Oliva Virgen ha de ser entrenado y formado para realizar los análisis organolépticos de las muestras de aceites de oliva virgen extra y 
virgen, y otros productos agroalimentarios de interés socioeconómico en la Cuenca Mediterránea.

El panel de catadores está constituido por personas que han sido seleccionadas por poseer cierta sensibilidad olfato gustativa y que están formadas en esta disciplina, para tener la capacidad de identificar sutiles diferencias entre los alimentos, son capaces de evaluarlos objetivamente. La función del panel de catadores expertos es fundamental para analizar que productos son los de más aceptación en el mercado, y van a ser más elegidos por los consumidores (Bagiani, 2020). Por tanto, un panel de catadores experto constituye una herramienta crucial para guiar en la toma de decisiones de las empresas de manera rápida y eficaz. El análisis sensorial proporciona también información de gran utilidad en investigación y determinación de olores y/o sabores no adecuados en alimentos, estudios de vida útil, etc.

Los paneles de cata deben entrenarse, estar validados según la legislación vigente (norma UNE-EN ISO 8586, ISO 5492, ISO 6658) y liderados por un jefe y subjefe de panel, con cualificación específica para ello. El Panel de Cata es una herramienta clave en el desarrollo de productos de valor añadido en la industria agroalimentaria, para asegurar el éxito en el lanzamiento de productos al mercado.

\section{Resultados}

La sociedad demanda una alimentación saludable y una adecuada dieta según las necesidades individuales, por tanto, formarse en este sector es prioritario, y con grandes oportunidades de futuro. Las campañas divulgativas de educación e información constituyen una importante herramienta para hacer que las informaciones sobre alimentos sean más comprensibles para los consumidores. El nivel de exigencia del mercado y las nuevas normativas de productos certificados de calidad hace que a los sectores agroalimentarios y de la restauración a tener más en cuenta las propiedades organolépticas. La sala de Cata de la Universidad de Granada entrena a estudiantes y a personas interesadas en el sector, para obtener una formación completa en esta disciplina del análisis sensorial. La sala, ubicada en la Facultad de Farmacia de la UGR, consta de todos los elementos y equipos necesarios para que pueda considerarse como una de las principales y, casi única, en nuestra región. Asimismo, constituye una plataforma de difusión y transferencia de conocimiento para el análisis del recetario tradicional granadino y andaluz, otorgándole un reconocimiento oficial que recupere y ponga en valor, las recetas típicas que conforman lo que podría denominarse como "Patrimonio Gastro-enológico con Denominación de Origen".

\section{Referencias}

Bigiani A (2020). Does ENaC work as sodium taste receptor in humans? Nutrients, $12 \quad$ (4), 1195. https://doi.org/10.3390/nu12041195

ISO 5492:2008. Análisis sensorial. Vocabulario. https://www.iso.org/obp/ui\#iso:std:iso:5492:ed-2:v1:amd:1:v1:es

ISO 6658:2019. Análisis sensorial. Metodología. Guía general. https://www.une.org/encuentra-tu-norma/busca-tunorma/norma?c=N0061499

ISO 8589:2010. Análisis sensorial. Guía general para el diseño de una sala de cata. https://www.une.org/encuentra-tu-norma/buscatu-norma/norma/?c=N0045203
Real Decreto 227/2008, de 15 de febrero, por el que se establece la normativa básica referente a los paneles de catadores de aceite de oliva virgen.

Pallante L, et al. (2021). On the human taste perception: Molecularlevel understanding empowered by computational methods. Trends in food science \& technology, 116: 445-459.

VIRTUOUS Project H2020-MSCA-RISE-2019. Referencia 872181. https://www.virtuoush2020.com

VIRTUOUS Project Proyecto de excelencia FEDER-Junta de $\begin{array}{lll}\text { Andalucia } & 2020 . & \text { Ref. }\end{array}$ https://www.juntadeandalucia.es/organismos/transformacionecono micaindustriaconocimientoyuniversidades/consejeria/transparencia /planificacion-evaluacion-estadistica/planes/detalle/86940.html 